\title{
Prophylactic Antibiotic Use in COPD and the Potential Anti-Inflammatory Activities of Antibiotics
}

\author{
Anthony W Huckle, Lucy C Fairclough MSc PhD, and Ian Todd MA PhD
}

\author{
Introduction \\ Do Antibiotics Have a Positive, Therapeutic Effect in Patients With Stable \\ COPD? \\ Azithromycin \\ Erythromycin \\ Clarithromycin \\ Roxithromycin \\ Doxycycline \\ Moxifloxacin \\ If Antibiotics Do Have a Positive, Therapeutic Effect in Patients With \\ COPD, Is This Potentially Anti-Inflammatory in Nature? \\ Effects on Inflammatory Cells \\ Effects on Transcription Factors \\ Effects on Cytokines and Inflammatory Mediators \\ Summary
}

\begin{abstract}
Antibiotics have previously demonstrated anti-inflammatory properties, and they have been linked to therapeutic benefit in several pulmonary conditions that feature inflammation. Previous research suggests that these anti-inflammatory properties may be beneficial in the treatment of COPD. This review assesses the potential benefit of prophylactic, long-term, and low-dose antibiotic therapy in COPD, and whether any effects seen are anti-inflammatory in nature. Randomized, controlled trials comparing antibiotic therapy with placebo in subjects with stable COPD were evaluated. Twelve trials involving 3,784 participants and a range of antibiotics were included: azithromycin (6 studies, 1,972 participants), clarithromycin (1 study, 67 participants), erythromycin (3 studies, 254 participants), roxithromycin (1 study, 191 participants), and moxifloxacin ( 2 studies, 1,198 participants). In vitro, in vivo, and ex vivo experimental study designs exploring the mechanisms via which antibiotics may act in subjects with stable COPD were evaluated. Azithromycin and erythromycin showed the greatest effect in subjects with COPD, with evidence suggesting improvement in exacerbation-related outcomes and health status, as measured by the St George Respiratory Questionnaire. An increase in antibiotic resistance was reported in 2 studies. The macrolide class of antibiotics exhibited convincing anti-inflammatory properties with relevance to COPD, implicating several pathways as potential mechanisms of action. In conclusion, the therapeutic benefit of macrolide antibiotics in subjects with stable COPD is consistent with anti-inflammatory properties, and macrolides should be considered as a potential therapy in COPD. Safety concerns regarding antibiotic resistance need to be addressed before widespread use in clinical practice. Key words: antibiotic; anti-inflammatory; azithromycin; anti-microbial; COPD; erythromycin; fluoroquinolone; immunomodulatory; macrolide. [Respir Care 2018;63(5):609-619. (C) 2018 Daedalus Enterprises]
\end{abstract}




\section{Prophylactic Antibiotics in COPD}

\section{Introduction}

COPD is defined by the Global Initiative for Chronic Obstructive Lung Disease (GOLD) ${ }^{1}$ as "a common, preventable, and treatable disease that is characterized by persistent respiratory symptoms and air-flow limitation that is due to airway and/or alveolar abnormalities usually caused by significant exposure to noxious particles or gases." COPD should be considered in patients with dyspnea, sputum production, chronic cough, and a history of risk factors for the disease. ${ }^{1}$ Spirometry is necessary for clinical confirmation; a post-bronchodilator $\mathrm{FEV}_{1} / \mathrm{FVC}<0.70$ is used to confirm diagnosis. Disease severity is assessed using post-bronchodilator $\mathrm{FEV}_{1}$ (GOLD Grades I-IV) and takes into account the modified Medical Research Council dyspnea scale, the COPD assessment test, and exacerbation frequency (grades A-D in increasing severity).

Chronic inflammation is key in the pathogenesis of COPD. Patients in a stable condition (ie, not currently experiencing an exacerbation) have a degree of underlying inflammation that contributes to reduced air flow. Macrophages and neutrophils are a significant cause of pulmonary damage in COPD. Chemo-attractants are released from macrophages and pulmonary epithelial cells, attracting an excess of neutrophils to the area. ${ }^{2}$ Subsequent release of metalloproteinases from these leukocytes causes extracellular matrix degradation ${ }^{3}$ and release of pro-inflammatory molecules, such as damage-associated molecular patterns.

Treatment for COPD is currently limited, based on lifestyle changes, use of bronchodilators and steroids, and antibiotic treatment for infectious exacerbations. Corticosteroids are of modest benefit in exacerbations, but they do little to reduce chronic inflammation. ${ }^{4}$ Hence, there is high demand for new adjuncts to treatment. Researchers are trying to find an effective treatment to target chronic inflammation, as it is hoped that reducing inflammation will lead to an improved quality of life for patients and possibly reduce exacerbation frequency, thus reducing mortality.

Mr Huckle and Drs Fairclough and Todd are affiliated with the School of Life Sciences, University of Nottingham, Nottingham, UK.

The authors have disclosed no conflicts of interest.

Supplementary material related to this paper is available at http:// www.rcjournal.com.

Correspondence: Lucy C Fairclough MSc PhD, School of Life Sciences, University of Nottingham, Life Sciences Building, University Park, Nottingham NG7 2RD, UK. E-mail: lucy.fairclough@nottingham.ac.uk.

DOI: $10.4187 /$ respcare. 05943
Antibiotics have been recognized to have anti-inflammatory effects beyond their antimicrobial activity. An early report of these immunomodulatory properties was a case in which erythromycin, a macrolide, had a therapeutic effect in diffuse panbronchiolitis. ${ }^{5}$ A follow-up study showed marked improvement in survival rates for diffuse panbronchiolitis after low-dose erythromycin therapy was introduced: the 5-y survival for subjects, which was $72 \%$ between 1980 and 1984, improved to $>90 \%$ after $1984 .{ }^{6}$ The possibility that other antibiotics may have similar effects in other pulmonary conditions, including COPD, has since been explored.

Current research suggests that certain antibiotics have the potential to reduce exacerbations in COPD patients who are at an increased exacerbation risk, with a 2013 review by Herath and Poole ${ }^{7}$ citing 7 studies using a range of macrolides (azithromycin, clarithromycin, erythromycin) and a fluoroquinolone (moxifloxacin); it was not possible to determine whether the antibiotics had a primarily antimicrobial or anti-inflammatory effect.

This review has 2 primary goals. First, we look at the question of whether the use of antibiotics in patients with stable COPD reduces exacerbation frequency and improves patient health status, acting as a qualitative update to the review of Herath and Poole in 2013. ${ }^{7}$ Second, we examine whether any effect seen can be attributed to the anti-inflammatory properties of antibiotics. We then discuss the ramifications of results on future research and clinical practice. The literature search strategies used for this review are detailed in the supplementary material (see the supplementary materials at http://www.rcjournal.com).

\section{Do Antibiotics Have a Positive, Therapeutic Effect in Patients With Stable COPD?}

A total of 12 randomized control trials, involving 3,692 subjects with COPD, have been included (Table 1). The results are discussed according to antibiotic. Of the 6 different antibiotics studied, 4 were macrolides: azithromycin, ${ }^{8-13}$ clarithromycin, ${ }^{14}$ erythromycin, ${ }^{15-17}$ and roxithromycin. ${ }^{18}$ One tetracycline, doxycycline, ${ }^{10}$ was used. One fluoroquinolone, moxifloxacin, ${ }^{10,19}$ was used. Only 1 study looked at a combination treatment (roxithromycin and doxycycline). ${ }^{18}$ Three of the 12 studies used pulsed antibiotic therapy..$^{10,12,19}$ Sethi et $\mathrm{al}^{19}$ and Brill et al ${ }^{10}$ used pulsed moxifloxacin, whereas Mygind et al ${ }^{12}$ used pulsed azithromycin. The remaining 9 studies used continuous therapy. A brief description of the studies used can be found in Supplementary Table 1.

\section{Azithromycin}

Azithromycin is a 15-membered macrolide studied in 6 of the 12 trials (Table 1). Five of the trials used continuous 
Table 1. Summary of Exacerbation-Related Outcomes Antibiotics Trials in COPD

\begin{tabular}{|c|c|c|c|}
\hline Antibiotic & $\begin{array}{l}\text { Total Studies } \\
\text { Included }\end{array}$ & Positive Outcome & Inconclusive Outcome \\
\hline Azithromycin & 6 & $\begin{array}{l}3 \text { studies }^{8,12,13} \text { showed a significant improvement } \\
\text { in exacerbation-related outcomes }\end{array}$ & $\begin{array}{l}3 \text { studies }^{9-11} \text { showed no significant improvement in } \\
\text { exacerbation-related outcomes, although } 2 \text { studies } \\
\text { showed improvement that did not reach } \\
\text { significance }\end{array}$ \\
\hline Erythromycin & 3 & $\begin{array}{l}3 \text { studies }{ }^{15-17} \text { showed a significant improvement } \\
\text { in exacerbation-related outcomes }\end{array}$ & NA \\
\hline Clarithromycin & 1 & NA & $\begin{array}{l}1 \text { study }^{14} \text { showed no significant improvement in } \\
\text { exacerbation-related outcomes }\end{array}$ \\
\hline Roxithromycin & 1 & NA & $\begin{array}{l}1 \text { study }^{18} \text { showed no significant improvement in } \\
\text { exacerbation-related outcomes }\end{array}$ \\
\hline Doxycycline & 2 & NA & $\begin{array}{l}2 \text { studies }{ }^{10,18} \text { showed no significant improvement in } \\
\text { exacerbation-related outcomes; Brill et a } 1^{10} \\
\text { showed a non-significant worsening exacerbation- } \\
\text { related outcomes } \\
\text { Note: Shafuddin et al }{ }^{18} \text { used roxithromycin }+ \\
\text { doxycycline }\end{array}$ \\
\hline Moxifloxacin & 2 & NA & $\begin{array}{l}2 \text { studies }^{10,19} \text { showed no significant improvement in } \\
\text { exacerbation-related outcomes, although Sethi } \\
\text { et } \mathrm{al}^{19} \text { showed a non-significant improvement }\end{array}$ \\
\hline
\end{tabular}

azithromycin therapy, and one used pulsed therapy. Four continuous trials used a dose of $250 \mathrm{mg}$, either daily or 3 times weekly. The other continuous trial used $500 \mathrm{mg} 3$ times weekly. The pulsed trial used $500 \mathrm{mg}$ for $3 \mathrm{~d} / \mathrm{month}$.

The study by Albert et $\mathrm{al}^{8}$ was the largest and most detailed, including 1,142 participants with GOLD stage II or worse. A group of 570 subjects was allocated to a treatment plan of $250 \mathrm{mg}$ azithromycin daily for 12 months, and this group was compared to 572 controls. The primary outcome was the time to the first exacerbation, and results showed a significant improvement for the azithromycin treatment group, $266 \mathrm{~d}$ compared to $174 \mathrm{~d}$ in the placebo group $(P<.001)$. Health status was also recorded using the St George Respiratory Questionnaire, with a lower score equating to better quality of life. The subjects treated with azithromycin had a significantly greater mean change in St George Respiratory Questionnaire score than the placebo group, $-2.8 \pm 12.1$ units compared to $-0.6 \pm 11.4$ units, respectively $(P=.006)$. It is important to note that while the azithromycin group did not see a change $>-4$ units, which is the minimally clinically important difference, there were significantly more subjects in the treatment group with a decrease of at least 4 units (43\%) than in the placebo group $(36 \%, P=.03)$.

The study by Mygind et al $^{12}$ was presented at the 2010 European Respiratory Society Congress. The study included 575 subjects with COPD, who were treated with $500 \mathrm{mg}$ azithromycin $3 \mathrm{~d} /$ month for 36 months. The full data have not been published, but a significant reduction in duration of exacerbation compared to placebo was reported.
There was no change in lung function, quality of life, or frequency of exacerbations.

Uzun et al $^{13}$ recruited 92 participants with GOLD-confirmed COPD, treating 47 of them with $500 \mathrm{mg} 3$ times weekly for 12 months and comparing them with 45 control subjects. The study aim was to investigate the rate of exacerbations in the first year of treatment. There was a lower exacerbation rate for the treatment group compared to the placebo group (1.94 vs 3.22, respectively), and the risk ratio showed a $42 \%$ decrease in exacerbations for the azithromycin group compared to control $(P=.001)$. A significant increase in median time to first exacerbation was also seen: $59 \mathrm{~d}$ in the placebo group compared to $130 \mathrm{~d}$ in the treatment group $(P=.001)$. In addition, exacerbation treatment required significantly fewer additional antibiotics in the azithromycin group than in the placebo group. An improvement in health status as measured with the St George Respiratory Questionnaire was reported at 3 months in favor of azithromycin $(P=.043)$, although this improvement did not remain for the full 12 months.

The final 3 studies used similar treatment patterns. Berkhof et $\mathrm{al}^{9}$ recruited 84 subjects with the primary aim of investigating cough-specific health status using the Leicester Cough Questionnaire. ${ }^{20}$ A group of 42 subjects was allocated to a treatment plan of $250 \mathrm{mg}$ azithromycin 3 times weekly for 12 weeks and compared to 42 controls. Berkhof et $\mathrm{al}^{9}$ showed significant improvements in health status for the azithromycin group using both the St George Respiratory Questionnaire and the Short Form 36. The St 
George Respiratory Questionnaire score difference between the 2 groups was $-7.5 \pm 2.5$ in favor of the treatment group, which is clinically important $(P=.004)$. Similarly, the Short Form 36 showed improvements in several domains in favor of treatment, including a difference of $8.3 \pm 3.4$ in "general health" between azithromycin and placebo $(P=.02)$. Further analysis showed that subjects with a lower baseline Leicester Cough Questionnaire score showed a greater improvement in both St George Respiratory Questionnaire and Short Form 36. This is an important finding and may indicate use of the Leicester Cough Questionnaire to identify COPD patients who are most likely to benefit from additional azithromycin therapy. Berkhof et $\mathrm{al}^{9}$ did not power their study to investigate exacerbation frequency, and there was not a significant difference between the groups in number of exacerbations. However, the data did show a non-significant decreased number of exacerbations.

Simpson et al ${ }^{11}$ recruited only 30 participants, and that study was powered to detect changes in the neutrophil chemokine CXCL8. A group of 15 subjects was allocated to a treatment plan of $250 \mathrm{mg}$ azithromycin daily for 12 weeks and compared to 15 controls. The mean rate of severe exacerbation was calculated and showed a nonsignificant decreased exacerbations in the treatment group: 0.33 exacerbations per person per 26 weeks compared to 0.93 in the placebo group $(P=.062)$.

Brill et al ${ }^{10}$ recruited 99 participants, splitting them over 3 different treatment groups, including a group treated with $250 \mathrm{mg}$ azithromycin 3 times weekly for 13 weeks. A group of 25 subjects was allocated to the treatment plan and compared to 24 controls. This study was not sufficiently powered to draw conclusions on changes in bacterial load. No significant improvement was seen in exacerbation rate or health status.

Three of the 6 studies found no significant adverse effects, ${ }^{9,11,13}$ and one did not report data. ${ }^{12}$ Both Brill et al ${ }^{10}$ and Albert et $\mathrm{al}^{8}$ reported significant adverse effects due to the azithromycin therapy. Albert et $\mathrm{al}^{8}$ reported a small but significant increase in hearing loss in the azithromycin group ( $25 \%$ vs $20 \%, P=.04)$. It is not clear whether this reversed when treatment was ended. It was also found that, although patients in the azithromycin group had decreased colonization with select respiratory pathogens, they also had an increase in colonization with macrolide-resistant organisms. Brill et $\mathrm{al}^{10}$ reported similar findings and showed a significant increase in antibiotic resistance with azithromycin treatment.

Together, these 6 studies show promising evidence that azithromycin as additional therapy in patients with stable COPD may reduce the rate of exacerbations and improve health status. Further large-scale trials that are sufficiently powered to investigate exacerbation rates are required, as are trials that compare pulsed versus continuous usage. If treatment is recommended, patients need to be monitored for the development of macrolide-resistant colonies.

\section{Erythromycin}

Erythromycin is a 14-membered macrolide studied in 3 of the 12 trials (Table 1). All 3 of these trials were included in a 2013 Cochrane Review, ${ }^{7}$ and no more recent trials fitting the criteria were found in the literature search. A study published in 2001 recruited 109 subjects to examine whether daily erythromycin (200-400 mg) for 12 months would reduce the frequency of exacerbations and common colds. ${ }^{17}$ A group of 55 subjects was allocated to the treatment group and compared to 54 controls. A limitation of this study was the lack of blinding, which had potential to influence results. The researchers showed a significant decrease in the number of exacerbations in the 12-month study period in the erythromycin group compared to placebo (14 vs. 64 , respectively, $P<.001$ ). An increased relative risk of experiencing one or more exacerbations was calculated for the control group to be $4.71(P=.007)$.

A later trial recruited 109 participants to investigate the number of moderate and severe exacerbations. ${ }^{16}$ A group of 53 subjects was allocated to a treatment plan of $250 \mathrm{mg}$ erythromycin twice daily for 12 months and compared to 56 controls. Three significant changes were seen: a decrease in exacerbation frequency was seen in the treatment arm; there was an increase in the median time to the first exacerbation in favor of the treatment group ( $271 \mathrm{~d}$ compared to $89 \mathrm{~d}, P=.02$ ); and there was a decrease in the duration of exacerbation for those on erythromycin therapy, with the average exacerbation lasting $13 \mathrm{~d}$ in the placebo arm versus $9 \mathrm{~d}$ in the treatment $\operatorname{arm}(P=.036)$.

$\mathrm{He}$ et $\mathrm{al}^{15}$ recruited 36 participants to investigate health status and the number of moderate and severe exacerbations. A group of 18 subjects was allocated to a treatment plan of $125 \mathrm{mg}$ erythromycin 3 times daily for 6 months and compared to 18 controls. As seen in the previous study, the median time to first exacerbation was increased in the treatment group compared to placebo, $155 \mathrm{~d}$ and $86 \mathrm{~d}$, respectively $(P=.032)$. Subjects in the erythromycin group also had a lower mean number of exacerbations over 6 months: 0.61 compared to 1.11 in the control group. $(P=.042)$. No significant improvements were seen in health status.

None of the above studies reported significant adverse effects. These 3 studies provide promising evidence for the use of erythromycin at a continuous low dose to reduce the frequency and duration of exacerbations. The evidence supports usage in patients with frequent exacerbations, but, as with azithromycin, patients should be monitored for macrolideresistant bacteria. 


\section{Clarithromycin}

Like erythromycin, clarithromycin is a 14-membered macrolide. The use of clarithromycin in subjects with stable COPD was investigated by Banerjee et al, ${ }^{14}$ who recruited 67 subjects for a 3-month trial (Table 1). A group of 31 subjects was allocated to a treatment plan of $500 \mathrm{mg}$ clarithromycin daily for 3 months and compared to 36 controls. Primary outcome was the health status score, which showed no significant improvement for the treatment group compared to the placebo group. No significant improvement was recorded in shuttle walk distance, spirometry, or C-reactive protein levels. The 2 groups showed no significant difference in exacerbation rate or development of multi-resistant colonies, however the study was not powered for these outcomes. The evidence is inconclusive, and there is no evidence to support clarithromycin usage in this patient group. Further research is necessary to make conclusions.

\section{Roxithromycin}

Roxithromycin is another 14-membered macrolide antibiotic. Shafuddin et al ${ }^{18}$ administered roxithromycin to 97 subjects at $300 \mathrm{mg}$ daily for 12 weeks, followed by a 48-week observation period (Table 1). The results were compared to 94 control subjects. No significant difference in exacerbation rate was seen between the roxithromycin or placebo groups in the 12-week treatment period or the 48-week observation period. Based on the results of this study, prophylactic roxithromycin use cannot be justified in patients with stable COPD, though further research may be warranted.

\section{Doxycycline}

Two recent studies examine the tetracycline antibiotic, doxycycline (Table 1). Brill et al ${ }^{10}$ recruited 99 subjects in total, splitting them over 3 different treatment groups including one with $100 \mathrm{mg}$ doxycycline daily for 13 weeks. Twenty-five subjects were allocated to the treatment plan and were compared to 24 controls. The study was insufficiently powered to detect a difference in bacterial load, this reduced the likelihood of recording significant outcomes. No significant differences between the doxycycline group and the placebo group were seen in any of the measured end points, apart from bacterial resistance, which increased by a factor of $3.74(P=.01)$. There was an unexpected non-significant increased exacerbations in the doxycycline group, with an increased adjusted relative risk of $2.07(0.99-4.35)(P=.05)$.

Shafuddin et al ${ }^{18}$ recruited 292 subjects, allocating 101 to receive a combination treatment of $300 \mathrm{mg}$ roxithromycin $+100 \mathrm{mg}$ doxycycline daily for 12 weeks and 94 subjects to act as a control. This study yielded no significant improvement in exacerbation frequency in either the 12-week treatment period or the 48-week observation period.

Neither study reported significant adverse events. The currently available data show no evidence of an effect in the use of prophylactic doxycycline treatment in COPD to prevent exacerbations. Further research is warranted.

\section{Moxifloxacin}

Moxifloxacin is a fluoroquinolone antibiotic; its effect in subjects with stable COPD has been examined in 2 recent studies (Table 1). A pulsed treatment plan was used in both trials of the drug. In the first trial, Sethi et al ${ }^{19}$ involved 1,149 subjects: 573 subjects were allocated to a treatment plan of $400 \mathrm{mg}$ moxifloxacin daily for $5 \mathrm{~d}$ every 8 weeks, for a total of 6 courses; 584 subjects acted as controls. The study gave both per-protocol and intentionto-treat data; only the intention-to-treat data are considered here. The primary outcome measure was the frequency of exacerbations, and on the basis of this definition, there was a non-significant decreased exacerbations in the moxifloxacin group (ie, a reduction of $19 \%, P=.059$ ). There was also a non-significant a longer time until the first exacerbation for the treatment group $(P=.062)$. Both treatment and control groups recorded an improvement in health status as measured by the St George Respiratory Questionnaire, although this improvement was not significant. The symptom domain of the St George Respiratory Questionnaire did show a significant improvement in the number of subjects with a decrease of at least 4 points (the minimum clinically important difference) in favor of moxifloxacin $(P=.01)$. With relevance to bacteriology, the mean minimum inhibitory concentration for several relevant bacteria changed very little throughout the study for both the treatment and control groups, indicating no development of bacterial resistance. There was a significant level of drug-related adverse events in the moxifloxacin group, with a large proportion of gastrointestinal disorders.

In the second trial, Brill et al ${ }^{10}$ involved 99 subjects, of whom 25 were allocated to receive treatment with $400 \mathrm{mg}$ moxifloxacin daily for $5 \mathrm{~d}$ every 4 weeks, for a total of 13 weeks; 24 subjects were allocated to a control group. This trial found no significant change in the exacerbation frequency of the moxifloxacin group; however, as mentioned earlier for the azithromycin subgroup, this study was not adequately powered for this outcome. One significant result was an increase in bacterial resistance to treatment, with a factor increase of 4.82 in minimum inhibitory concentration $(P=.01)$.

These 2 studies are too small to draw reliable conclusions, and additional high-powered studies are necessary 
to determine whether pulsed moxifloxacin treatment can be of use in patients with stable COPD for exacerbation prevention.

\section{If Antibiotics Do Have a Positive, Therapeutic Effect in Patients With COPD, Is This Potentially Anti-Inflammatory in Nature?}

Finding a way to effectively manage the chronic inflammation in COPD may be key in reducing mortality and slowing disease progression. Each subsequent GOLD stage has been shown to be associated with more severe thickening of the airway wall and increased polymorphonuclear neutrophil and macrophage infiltration. ${ }^{21}$ Corticosteroids are often used to target general inflammation, but they have only a modest effect on airway inflammation in COPD. This is thought to be due to reduced histone deacetylase (HDAC) activity in COPD patients. ${ }^{22}$ Macrolides and other classes of antibiotics have therefore attracted attention as potential therapies in COPD due to the large body of evidence indicating that they display immunomodulatory effects. ${ }^{23-25}$ The research discussed in the previous section provides strong support for the use of certain antibiotics as long-term therapy in stable COPD, particularly azithromycin and erythromycin, although moxifloxacin showed potential benefit as well. A major risk of long-term antibiotic use, however, is development of antibiotic resistance. Two of the studies listed above showed an increase in bacterial resistance due to treatment. ${ }^{8,10}$ If research can confirm that the therapeutic effect of antibiotics in patients with stable COPD is primarily anti-inflammatory in nature rather than antimicrobial, then non-antibiotic derivatives could be suitable alternatives (eg, EM900, EM703).26,27

This part of the review focuses on 18 studies that reported the effects of antibiotics on the number of inflammatory cells (neutrophils and macrophages), inflammatory cytokine levels (interleukin 1-beta [IL-1 $\beta$ ], IL-6, IL-8, IL10 , tumor necrosis factor alpha $[\mathrm{TNF}-\alpha]$, granulocytemacrophage colony-stimulating factor, CXC chemokines), or other inflammatory markers (eg, C-reactive protein). Of the 18 studies, only 4 used antibiotics that are not macrolides: Zimmerman et $\mathrm{al}^{28}$ and Schild et $\mathrm{al}^{29}$ used moxifloxacin; Siva et $\mathrm{al}^{30}$ and Zimmerman et $\mathrm{al}^{28}$ used levofloxacin; Prins used doxycycline ${ }^{31}$. The remaining studies, including Zimmerman et al, ${ }^{28}$ used macrolide antibiotics. This includes azithromycin, ${ }^{11,28,32-37}$ clarithromycin, ${ }^{35,38}$ erythromycin, ${ }^{15,16,35,39,40}$ roxithromycin, ${ }^{35}$ solithromycin, ${ }^{41}$ and the non-antibiotic azithromycin derivative CSY0073. ${ }^{42}$ A brief description of the studies used can be found in Supplementary Table 2.

\section{Effects on Inflammatory Cells}

As described earlier, neutrophils and macrophages have an important role in the initial immune response to tissue in- sult; in COPD, this may include reaction to the bioactive lipopolysaccharide found in cigarette smoke..$^{43}$ Reactive oxygen species are released from airway inflammatory cells and can lead to activation of transcription factors such as nuclear factor kappa B (NF- $\kappa \mathrm{B})$ as well as mitogen-associated protein kinases. ${ }^{44}$ Constant release of proinflammatory cytokines from macrophages and neutrophils prevents resolution and leads to a perpetual state of chronic inflammation. Some studies have also linked increased sputum neutrophil levels with a faster decline in $\mathrm{FEV}_{1}{ }^{45}$ Several of the above studies examined the effects of the antibiotics on the presence of inflammatory cells in the airways and the potential for use in patients with COPD.

A study published by Hodge et $\mathrm{al}^{32}$ surmised from previous research that ineffective clearance of apoptotic bronchial epithelial cells by alveolar macrophages can encourage inflammation, as necrotic material is not removed. It is possible that this is due to either an inability of the macrophages to deal with the excessive apoptosis, or a "functional deficiency" within the macrophages. Hodge et al $^{32}$ investigated whether low-dose azithromycin could improve the phagocytic function of alveolar macrophages. The research showed that baseline macrophage ability was lower in subjects with COPD $(P=.005)$, which is consistent with the previous theory that failed clearance of apoptotic products may contribute to inflammation. Bronchoalveolar lavage was collected from the subjects ( 10 healthy volunteers and 9 subjects with COPD), and samples were incubated with a stimulant in vitro. Azithromycin was the macrolide stimulant tested, and it was compared to clindamycin as a negative control and to granulocyte-macrophage colony-stimulating factor as a positive control. Results showed that azithromycin caused a significant increase in alveolar macrophages that had phagocytosed a bronchial epithelial cell (ie, a mean increase of $67 \%$ in the COPD population). A significant increase in the percentage of apoptotic neutrophils that had been phagocytosed was also noted. These effects were dependent on the duration of alveolar macrophage incubation with the antibiotic, rather than on the duration of bronchial epithelial cell incubation, indicating that azithromycin promotes increased phagocytosis. Another interesting finding was that phagocytosis was inhibited by phosphatidylserine lysosomes. This suggests that the mechanism of azithromycin's pro-phagocytic effects may be partially due to effects on the phosphatidylserine pathway, which promotes macrophage phagocytosis of epithelial cells.

A subsequent study by some of the same researchers produced similar results after treatment with azithromycin (ie, an improved ability of alveolar macrophages to phagocytose apoptotic bronchial epithelial cells). ${ }^{33}$ The researchers also investigated the role of the mannose receptor, which was shown to be significantly underexpressed by subjects with COPD. A link was demonstrated between 
the mannose receptor and the phagocytic capabilities of alveolar macrophages in vitro; blocking the mannose receptor caused a $60 \%$ reduction in alveolar macrophage phagocytosis of apoptotic cells. This is relevant as patients with COPD have a lower percentage of alveolar macrophages expressing the mannose receptor, which may help explain the ongoing inflammation in patients with COPD. Azithromycin treatment was shown by the researchers to significantly increase mannose receptor expression and thus increase phagocytosis. These results implicate defective mannose receptor expression as a contributing factor in COPD, leading to decreased alveolar macrophage phagocytosis. Neither of these 2 trials recorded neutrophil sputum levels. One study that did so found a nonsignificant reduction in sputum neutrophil proportion for subjects with COPD treated with azithromycin compared to control. ${ }^{11}$

Particularly relevant for COPD was a trial conducted on mice using a non-antibiotic azithromycin analog, CSY0073. ${ }^{42}$ CSY0073 significantly decreased the neutrophil count by $23 \%$ in lung inflammation induced by lipopolysaccharide challenge. This was not as large a decrease as with azithromycin, which reduced the count by $63 \%$, but it showed that a nonantibiotic macrolide can still have anti-inflammatory properties. This is important as excessive antibiotic usage in COPD can lead to microbial resistance.

Two of the above studies examined the possible effects of erythromycin on inflammatory cell count. The first, published in 2010, reported a significant reduction in the neutrophil cell count in sputum from baseline at 3 months and 6 months with low-dose erythromycin treatment compared to placebo. ${ }^{15}$ The second study, published in 2016, featured 2 treatment groups with a placebo control..$^{40}$ One treatment group received $125 \mathrm{mg} 3$ times daily for 6 months, as in the previous study, ${ }^{15}$ whereas the other received the same dosage for 12 months. A significant reduction in sputum neutrophil count was seen as early as 3 months into the trial and was mirrored by an increase in macrophage ratio, indicating a reduction in neutrophils and an increase in macrophage presence. These results suggest that erythromycin has a similar mechanism to azithromycin, as shown by Hodge et al, ${ }^{32}$ with involvement of the phosphatidylserine pathways and the mannose receptors of epithelial cells.

A mouse model study ${ }^{38}$ evaluated the efficacy of clarithromycin in preventing emphysema, a characteristic feature of COPD, in mice exposed to cigarette smoke extract. Six months of exposure to smoke led to a 2.3 -fold increase in macrophage accumulation in the lungs of mice exposed to smoke versus those not exposed $(P<.001)$. This confirms the role of cigarette smoke in initiating the inflammatory response. Clarithromycin treatment significantly inhibited this macrophage infiltration, with the treatment group showing an increase of $38.3 \pm 3.66 \times 10^{3}$ compared to the non-treatment group's increase of $56.5 \pm 4.39 \times 10^{3}$
$(P<.001)$. These results suggest clarithromycin has an antiinflammatory mechanism that reduces inflammation onset in mice exposed to smoke. Reduced alveolar macrophage infiltration may lead to reduced pro-inflammatory cytokine release and may reduce extracellular tissue destruction. It may be that other macrolide antibiotics have a similar mechanism and can partially account for their beneficial effects in stable COPD.

One study examining the impact of the fluoroquinolone levofloxacin investigated the effect on sputum neutrophil count in subjects with stable COPD. ${ }^{30}$ The treatment group showed only a minor reduction in sputum neutrophil count with levofloxacin. However, when limiting analysis to patients with a high baseline bacterial load $\left(>10^{6}\right.$ colonyforming units $/ \mathrm{mL}$ ), there was a significant reduction of $26.5 \%$ in the neutrophil count when compared to the placebo control.

\section{Effects on Transcription Factors}

Transcriptional factors (eg, NF- $\kappa \mathrm{B})$ can induce numerous pro-inflammatory processes and molecules, such as chemokines, adhesion molecules, and angiogenic factors. Cigarette smoke is an important risk factor for increased NF- $\kappa \mathrm{B}$ activity, so it is likely that targeting NF- $\kappa \mathrm{B}$ is a viable mechanism of therapy to reduce chronic inflammation in COPD.

Erythromycin has been shown to reduce NF- $\kappa$ B activity in human monocytes that had been treated with cigarette smoke extract. ${ }^{39}$ Similarly, results showed decreased NF$\kappa \mathrm{B}-$ related protein expression in human macrophages. These results support the conclusion that erythromycin has an effect in reducing inflammation via modulation of NF- $\kappa \mathrm{B}$ activity. The expression of HDAC proteins was also measured in this study; although they are not transcriptional factors, they have an important role in attenuating inflammatory responses. Previous studies have shown that cigarette smoke extract reduces HDAC protein expression; ${ }^{46}$ $\mathrm{Li}$ et $\mathrm{al}^{39}$ confirmed this when they found that pretreating human macrophages with erythromycin increased protein expression of HDAC 1, 2, and 3, while it had no effect on HDAC activity. ${ }^{39} \mathrm{It}$ is possible that no significant increase was seen in HDAC activity due to too small a sample size, so further large trials are recommended. As discussed earlier, decreased HDAC activity is thought to partially explain the modest effects of corticosteroids as treatment. The positive results from this trial suggest erythromycin may have the potential to restore HDAC protein expression and thus improve corticosteroid efficacy in treating COPD inflammation.

A later study involved similar experiments in the macrophage cell line U937, with the aim of comparing solithromycin, a novel macrolide, with several other macrolides. ${ }^{41}$ The results showed that solithromycin treatment 
significantly inhibits NF- $\kappa \mathrm{B}$ activity, while the other macrolides (ie, azithromycin, clarithromycin, erythromycin, telithromycin) showed no significant results. The research offers no explanation for why erythromycin failed to show a reduction in NF- $\kappa \mathrm{B}$ activity in this trial; however, it does show that solithromycin has the potential to act as an anti-inflammatory agent in COPD by reducing NF- $\kappa \mathrm{B}$ activity.

One study investigated NF- $\kappa \mathrm{B}$ activation during lung inflammation in mice, using azithromycin as treatment. ${ }^{36}$ Unlike the previous study, Stellari et al ${ }^{36}$ showed azithromycin to inhibit in vivo NF- $\kappa \mathrm{B}$ activation in a dosagedependent pattern. This adds further evidence that certain macrolides can act as anti-inflammatory agents, although studies on human cell lines need to be conducted.

The 3 studies discussed above largely corroborate each other and suggest that azithromycin, erythromycin, and solithromycin all inhibit NF- $\kappa$ B expression and activation, which may be a potential mechanism by which pro-inflammatory cytokines in COPD can be modulated. Further studies would be of use, particularly those examining other classes of antibiotics (eg, fluoroquinolones).

\section{Effects on Cytokines and Inflammatory Mediators}

Table 2 shows the macrolide class of antibiotics to be the most effective at inhibiting pro-inflammatory interleukins (IL-1 $\beta$, IL-6, IL-10, IL-12, IL-13, IL-17, IL-23), chemokines (IL-8, CXCLs, CCLs), and other inflammatory cytokines (TNF- $\alpha$ and granulocyte-macrophage colony-stimulating factor). Azithromycin shows the most convincing evidence in support of anti-inflammatory properties: it was found to reduce levels of every inflammatory molecule it was tested on, across a range of study types. This included inhibiting IL-10 and IL-12, which none of the other antibiotics were shown to reduce. Clarithromycin and roxithromycin were both shown to have very similar immunomodulatory properties, ${ }^{35}$ so they may also be effective anti-inflammatory agents. In contrast, erythromycin did not affect cytokine or chemokine release.

The novel non-antibiotic macrolide CSY0073 had positive results and an anti-inflammatory profile similar to that of azithromycin. Novel macrolides such as CSY0073 have potential in COPD therapy because they do not inhibit bacterial growth and possess no antimicrobial properties, which theoretically makes them suitable for longerterm usage without causing microbial resistance. In one study, it was noted that withdrawal of therapy led to a return of cytokine levels to baseline, so prolonged therapy is desirable. ${ }^{40}$ The tetracycline antibiotic doxycycline showed poor results in the only study in which it was used because it had no effect on any of the inflammatory markers in sputum samples from subjects with COPD. Of the 2 fluoroquinolones used in the studies above, moxifloxacin and levofloxacin, moxifloxacin appeared to be the superior, although both showed poor results.

\section{Summary}

This review indicates that prophylactic use of certain low-dose macrolide antibiotics is of benefit in reducing exacerbation-related outcomes in COPD. These include reducing exacerbation frequency, duration of exacerbation, and the time until first exacerbation after starting treatment. This conclusion applies only to azithromycin and erythromycin, as the other antibiotics showed no clinically important improvement, and no evidence of effect could be shown from the available studies. This could be due to a lack of therapeutic effect from the other antibiotics studied, but is more likely due to a dearth of sufficiently powered studies investigating relevant outcomes.

Analysis of whether antibiotics have an anti-inflammatory role in COPD identified some possible mechanisms by which antibiotics can alter the pulmonary environment. Azithromycin was shown to have important effects on inflammatory cells, including improving phagocytic function of alveolar macrophages, up-regulation of the phosphatidylserine pathway, and increased expression of mannose receptors on alveolar macrophages. Macrolides have been shown to accumulate in high concentrations in alveolar macrophages compared to other classes of antibiotics. ${ }^{47-49}$ This may partially explain their efficacy in altering alveolar macrophage activity.

Macrolides were also shown to have effects on transcriptional factors and mediator molecules. Erythromycin restored HDAC protein expression, which supports the findings of previous research, ${ }^{46}$ and azithromycin, solithromycin, and erythromycin all appear to have a role in reducing NF- $\kappa \mathrm{B}$ activity. The restoration of HDAC expression is an important finding because research has suggested that reduced HDAC activity is a reason why corticosteroids have only modest effects on chronic inflammation in COPD;22 therefore, erythromycin therapy may improve corticosteroid efficacy.

Azithromycin, clarithromycin, and roxithromycin were shown to have the broadest range of effects on pro-inflammatory cytokine production. Erythromycin failed to show a strong ability to inhibit cytokines and chemokines, which is not consistent with the results from the other macrolide antibiotics. This may suggest that erythromycin and azithromycin have different mechanisms of action; while both provide benefits to patients with COPD, they do not share the same anti-inflammatory profile. However, this requires confirmation because much of the cytokine and chemokine data on macrolides came from a single study. ${ }^{35}$ Doxycycline, moxifloxacin, and levofloxacin were not studied in depth regarding their anti-inflammatory effects, so there 
Table 2. Summary of Modulatory Effects of Antibiotics in COPD

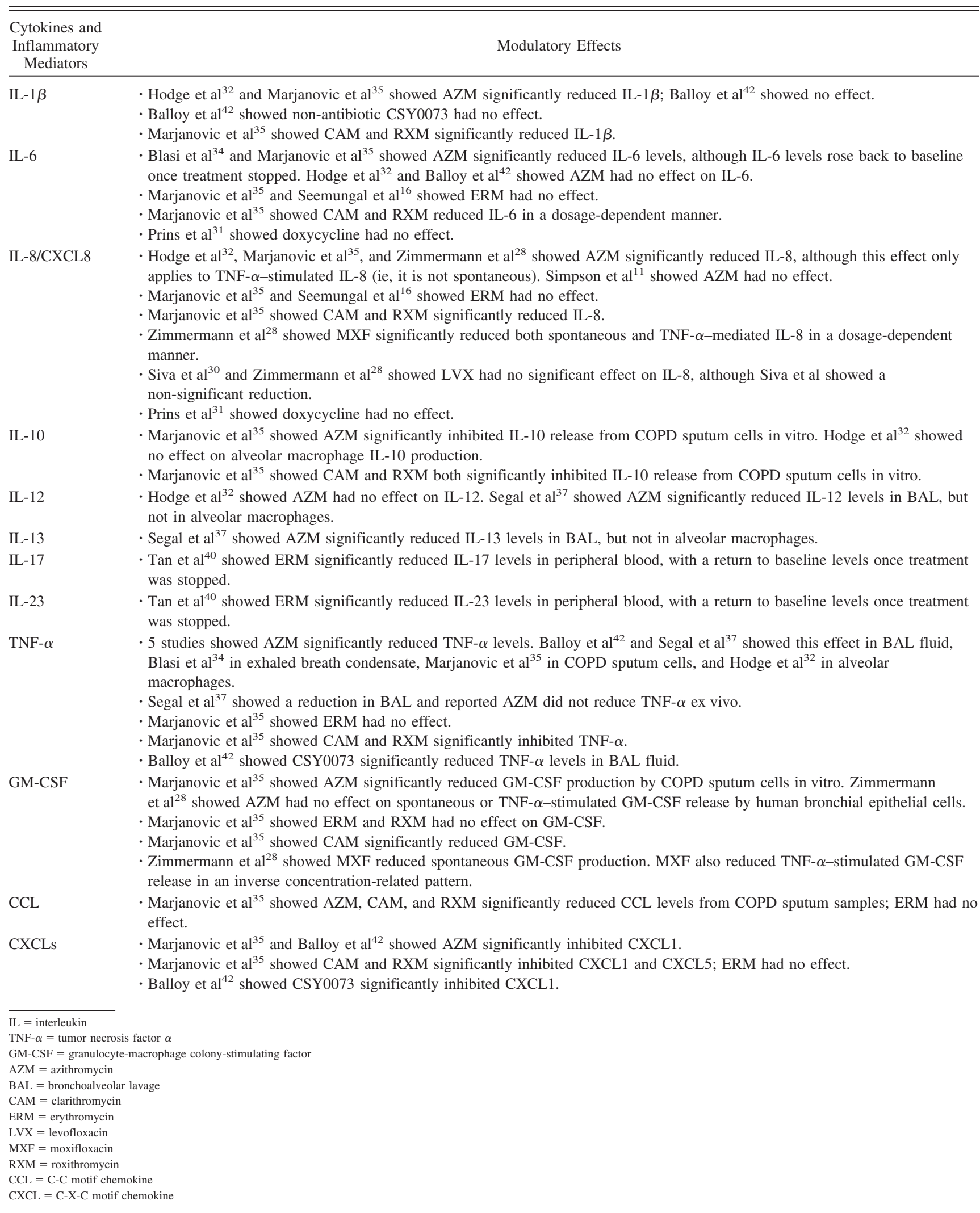




\section{Prophylactic Antibiotics in COPD}

is insufficient evidence to draw conclusions. Overall, the evidence is consistent with the macrolide health benefits in COPD being, at least partly, due to anti-inflammatory properties.

Appropriate conclusions have been made on the basis of available evidence for azithromycin and erythromycin, but the evidence is incomplete regarding clarithromycin, roxithromycin, and the fluoroquinolone class of antibiotics. The conclusions made above are not necessarily applicable to the wider COPD population. Eleven out of 12 studies reported full data on the subjects included; the subjects were typically older than the average COPD patient, and subjects with relevant comorbidities, such as prolonged QT interval, resting tachycardia, or other respiratory pathologies (eg, asthma, pulmonary cancer), were often excluded. This reduces the generalizability of the results because many patients with COPD have comorbidities, so care needs to be taken to restrict macrolide usage to suitable patients.

In conclusion, this review supports the use of continuous low-dose azithromycin or erythromycin therapy to improve exacerbation-related COPD outcomes. Patients should be monitored for the development of antibiotic resistance, and care needs to be taken to avoid unnecessary usage. There is no clear evidence of effect for the other antibiotics discussed, so no conclusions can be drawn. Thus, the research, while convincing, is incomplete and further large-scale trials need to be conducted. Specifically, these trials should compare the macrolide antibiotics and various treatment plans and continuous therapy versus pulsed therapy. It is important for future studies to be adequately powered to investigate a range of exacerbationrelated outcomes, health status, and adverse events. Clinicians need this information to make the best decisions for patients.

In view of the strong evidence for anti-inflammatory effects of certain macrolide antibiotics of relevance to COPD, but bearing in mind the danger of bacterial resistance resulting from prolonged use of antibiotics, the development of non-antibiotic macrolide analogues (eg, CSY0073) should be a focus of further research. These molecules have important potential in COPD treatment, because they would enable long-term use without promoting antibiotic resistance.

\section{ACKNOWLEDGMENTS}

The authors thank Dr Kieran Ayling for advice on performing the review.

\section{REFERENCES}

1. Global Strategy for the Diagnosis, Management and Prevention of COPD. Global Initiative for Chronic Obstructive Lung Disease (GOLD), 2017. http://goldcopd.org. Accessed on 8 Dec 2017.
2. Hoenderdos K, Condliffe A. The neutrophil in chronic obstructive pulmonary disease: too little, too late or too much, too soon? Am J Respir Cell Mol Biol 2013;48(5):531-539.

3. Tubby C, Harrison T, Todd I, Fairclough L. Immunological basis of reversible and fixed airways disease. Clin Sci (Lond.) 2011;121(7): 285.

4. Roche N, Huchon G. Reducing airways inflammation to prevent exacerbations in chronic obstructive pulmonary disease. Allergy 2005; 60(11):1350-1356.

5. Kudoh S, Kimura H. Clinical effect of low-dose long-term administration of macrolides on diffuse panbronchiolitis. Japan J Thorac Dis 1984;22(Suppl.): 254 .

6. Kudoh S, Azuma A, Yamamoto M, Izumi T, Ando M. Improvement of survival in patients with diffuse panbronchiolitis treated with lowdose erythromycin. Am J Respir Crit Care Med 1998;157(6 Pt 1): 1829-1832.

7. Herath SC, Poole P. Prophylactic antibiotic therapy for chronic obstructive pulmonary disease (COPD). Cochrane Database Syst Rev 2013;11:CD009764.

8. Albert RK, Connett J, Bailey WC, Casaburi R, Cooper Jr JAD, Criner GJ, et al. Azithromycin for prevention of exacerbations of COPD. N Engl J Med 2011;365(8):689-698.

9. Berkhof FF, Doornewaard-ten Hertog NE, Uil SM, Kerstjens HA, van den Berg JW. Azithromycin and cough-specific health status in patients with chronic obstructive pulmonary disease and chronic cough: a randomised controlled trial. Respir Res 2013;14:125.

10. Brill SE, Law M, El-Emir E, Allinson JP, James P, Maddox V, et al. Effects of different antibiotic classes on airway bacteria in stable COPD using culture and molecular techniques: A randomised controlled trial. Thorax 2015;70(10):930-938.

11. Simpson JL, Powell H, Baines KJ, Milne D, Coxson HO, Hansbro $\mathrm{PM}$, et al. The effect of azithromycin in adults with stable neutrophilic COPD: A double blind randomised, placebo controlled trial. PLoS ONE 2014;9(8):e105609.

12. Mygind L, Torp-Pedersen C, Vestbo J, Christensen JJ, FrimodtMøller N, Kirstiansen IS. A randomised, placebo-controlled 3 years study of prophylactic azithromycin in 575 patients with chronic obstructive pulmonary disease. Eur Respir J 2010;36:A5580.

13. Uzun S, Djamin RS, Kluytmans JAJW, Mulder PGH, van't Veer NE, Ermens AAM, et al. Azithromycin maintenance treatment in patients with frequent exacerbations of chronic obstructive pulmonary disease (COLUMBUS): A randomised, double-blind, placebo-controlled trial. Lancet Respir Med 2014;2(5):361-368.

14. Banerjee D, Honeybourne D, Khair OA. The effect of oral clarithromycin on bronchial airway inflammation in moderate-to-severe stable COPD: a randomized controlled trial. Treat Respir Med 2004; 3(1):59-65.

15. He ZY, Ou LM, Zhang JQ, Bai J, Liu GN, Li MH, et al. Effect of 6 months of erythromycin treatment on inflammatory cells in induced sputum and exacerbations in chronic obstructive pulmonary disease. Respiration 2010;80(6):445-452.

16. Seemungal TAR, Wilkinson TMA, Hurst JR, Perera WR, Sapsford RJ, Wedzicha JA. Long-term erythromycin therapy is associated with decreased chronic obstructive pulmonary disease exacerbations. Am J Respir Crit Care Med 2008;178(11):1139-1147.

17. Suzuki T, Yanai M, Yamaya M, Satoh-Nakagawa T, Sekizawa K, Ishida $\mathrm{S}$, et al. Erythromycin and common cold in COPD. Chest 2001;120(3):730-733.

18. Shafuddin E, Mills GD, Holmes MD, Poole PJ, Mullins PR, Black PN. A double-blind, randomised, placebo-controlled study of roxithromycin and doxycycline combination, roxithromycin alone, or matching placebo for 12 weeks in adults with frequent exacerbations of chronic obstructive pulmonary disease. J Negat Results Biomed 2015;14:15. 


\section{Prophylactic Antibiotics in COPD}

19. Sethi S, Jones PW, Theron MS, Miravitlles M, Rubinstein E, Wedzicha JA, et al. Pulsed moxifloxacin for the prevention of exacerbations of chronic obstructive pulmonary disease: a randomized controlled trial. Respir Res 2010;11(1):10.

20. Berkhof FF, Boom LN, ten Hertog NE, Uil SM, Kerstjens HAM, van den Berg JWK. The validity and precision of the Leicester cough questionnaire in COPD patients with chronic cough. Health Qual Life Outcomes 2012;10:4.

21. Hogg JC, Chu F, Utokaparch S, Woods R, Elliott WM, Buzatu L, et al. The nature of small-airway obstruction in chronic obstructive pulmonary disease. N Engl J Med 2004;350(26):2645-2653.

22. Ito K, Ito M, Elliott WM, Cosio B, Caramori G, Kon OM, et al. Decreased histone deacetylase activity in chronic obstructive pulmonary disease. N Engl J Med 2005;352(19):1967-1976.

23. Sadarangani SP, Estes LL, Steckelberg JM. Non-anti-infective effects of antimicrobials and their clinical applications: a review. Mayo Clin Proc 2015;90(1):109-127.

24. Kudoh S. Erythromycin treatment in diffuse panbronchiolitis. Curr Opin Pulm Med 1998;4(2):116-121.

25. Altenburg J, de Graaff CS, van der Werf TS, Boersma WG. Immunomodulatory effects of macrolide antibiotics - part 1: biological mechanisms. Respiration 2011;81(1):67-74.

26. Sugawara A, Sueki A, Hirose T, Nagai K, Gouda H, Hirono S, et al. Novel 12-membered non-antibiotic macrolides from erythromycin A; EM900 series as novel leads for anti-inflammatory and/or immunomodulatory agents. Bioorg Med Chem Lett 2011;21(11):33733376.

27. Gouda H, Sunazuka T, Yoshida K, Sugawara A, Sakoh Y, Omura S, et al. Three-dimensional solution structure of EM703 with potent promoting activity of monocyte-to-macrophage differentiation. Bioorg Med Chem Lett 2006;16(9):2496-2499.

28. Zimmermann GS, Neurohr C, Villena-Hermoza H, Hatz R, Behr J. Anti-inflammatory effects of antibacterials on human Bronchial epithelial cells. Respir Res 2009;10:89.

29. Schild K, Knobloch J, Yakin Y, Jungck D, Urban K, Muller K, et al. IL-5 release of $\mathrm{CD} 4+$ non-effector lymphocytes is increased in COPD-modulating effects of moxifloxacin and dexamethasone. Int Immunopharmacol 2011;11(4):444-448.

30. Siva R, Bafadhel M, Monteiro W, Brightling CE, Pavord ID. Effect of levofloxacin on neutrophilic airway inflammation in stable COPD: a randomized, double-blind, placebo-controlled trial. Int J Chron Obstruct Pulmon Dis 2014;9:179-186.

31. Prins HJ, Daniels JM, Lindeman JH, Lutter R, Boersma WG. Effects of doxycycline on local and systemic inflammation in stable COPD patients, a randomized clinical trial. Respir Med 2016;110:46-52.

32. Hodge S, Hodge G, Brozyna S, Jersmann H, Holmes M, Reynolds PN. Azithromycin increases phagocytosis of apoptotic bronchial epithelial cells by alveolar macrophages. Eur Respir J 2006;28(3):486495.

33. Hodge S, Hodge G, Jersmann H, Matthews G, Ahern J, Holmes M, et al. Azithromycin improves macrophage phagocytic function and expression of mannose receptor in chronic obstructive pulmonary disease. Am J Respir Crit Care Med 2008;178(2):139-148.

34. Blasi F, Bonardi D, Aliberti S, Tarsia P, Confalonieri M, Amir O, et al. Long-term azithromycin use in patients with chronic obstructive pulmonary disease and tracheostomy. Pulm Pharmacol Ther 2010;23(3):200-207.
35. Marjanovic N, Bosnar M, Michielin F, Wille DR, Anic-Milic T, Culic O, et al. Macrolide antibiotics broadly and distinctively inhibit cytokine and chemokine production by COPD sputum cells in vitro. Pharmacol Res 2011;63(5):389-397.

36. Stellari FF, Caruso P, Topini T, Carnini C, Facchinetti F, Villetti G. Anti-inflammatory effects of azithromycin evaluated by in vivo imaging of NF-KB activation in a mouse model of acute lung inflammation. Am J Respir Crit Care Med 2012;185:A5585.

37. Segal LN, Clemente JC, Wu BG, Wikoff WR, Gao Z, Li Y, et al. Randomised, double-blind, placebo-controlled trial with azithromycin selects for anti-inflammatory microbial metabolites in the emphysematous lung. Thorax 2016.

38. Nakanishi Y, Kobayashi D, Asano Y, Sakurai T, Kashimura M, Okuyama S, et al. Clarithromycin prevents smoke-induced emphysema in mice. Am J Respir Crit Care Med 2009;179(4):271-278.

39. Li M, Zhong X, He Z, Wen M, Li J, Peng X, et al. Effect of erythromycin on cigarette-induced histone deacetylase protein expression and nuclear factor-kappaB activity in human macrophages in vitro. Int Immunopharmacol 2012;12(4):643-650.

40. Tan C, Huang H, Zhang J, He Z, Zhong X, Bai J. Effects of low-dose and long-term treatment with erythromycin on interleukin-17 and interleukin-23 in peripheral blood and induced sputum in patients with stable chronic obstructive pulmonary disease. Mediators Inflamm 2016;2016:4173962.

41. Kobayashi Y, Wada H, Rossios C, Takagi D, Higaki M, Mikura S, et al. A novel macrolide solithromycin exerts superior anti-inflammatory effect via NF-kappaB inhibition. J Pharmacol Exp Ther 2013; 345(1):76-84

42. Balloy V, Deveaux A, Lebeaux D, Tabary O, Le Rouzic P, Ghigo $\mathrm{JM}$, et al. Azithromycin analogue CSY0073 attenuates lung inflammation induced by LPS challenge. Br J Pharmacol 2014;171(7): 1783-1794.

43. Hasday JD, Bascom R, Costa JJ, Fitzgerald T, Dubin W. Bacterial endotoxin is an active component of cigarette smoke. Chest 1999; 115(3):829-835.

44. Rahman I. The role of oxidative stress in the pathogenesis of COPD: implications for therapy. Treat Respir Med 2005;4(3):175-200.

45. Stanescu D, Sanna A, Veriter C, Kostianev S, Calcagni PG, Fabbri LM, et al. Airways obstruction, chronic expectoration, and rapid decline of FEV1 in smokers are associated with increased levels of sputum neutrophils. Thorax 1996;51(3):267-271

46. Yang SR, Chida AS, Bauter MR, Shafiq N, Seweryniak K, Maggirwar SB, et al. Cigarette smoke induces proinflammatory cytokine release by activation of NF-kappaB and posttranslational modifications of histone deacetylase in macrophages. Am J Physiol Lung Cell Mol Physiol 2006;291(1):L46-L57.

47. Patel KB, Xuan D, Tessier PR, Russomanno JH, Quintiliani R, Nightingale $\mathrm{CH}$. Comparison of bronchopulmonary pharmacokinetics of clarithromycin and azithromycin. Antimicrob Agents Chemother 1996;40(10):2375-2379.

48. Rodvold KA, Danziger LH, Gotfried MH. Steady-state plasma and bronchopulmonary concentrations of intravenous levofloxacin and azithromycin in healthy adults. Antimicrob Agents Chemother 2003; 47(8):2450-2457.

49. Hand WL, Boozer RM, King-Thompson NL. Antibiotic uptake by alveolar macrophages of smokers. Antimicrob Agents Chemother 1985;27(1):42-45. 